Www.jmscr.igmpublication.org

Impact Factor (SJIF): 6.379

Index Copernicus Value: 79.54

ISSN (e)-2347-176x ISSN (p) 2455-0450

crossrefDOI: https://dx.doi.org/10.18535/jmscr/v6i10.130

Journal Of Medical Science And Clinical Research

\title{
The incidence of differentiated carcinoma thyroid as histopathologic surprise in FNAC proved benign thyroid nodules
}

\author{
Authors \\ Dr Nandagopal Vasudevan ${ }^{1}$, Dr Premlal A.P ${ }^{2}$, Dr Balagopal $\mathrm{K}^{3}$ \\ ${ }^{1}$ Consultant Urologist, Sree Uthradom Thirunal Hospital, Pattom, Thiruvananthapuram \\ ${ }^{2}$ Associate Professor, Department of Plastic Surgery, Govt. Medical college, Thiruvananthapuram, State \\ Nodal Officer, Trauma \& Burns, Kerala \\ ${ }^{3}$ Retd. Professor \& Head of Department of Surgery, Govt. Medical college, Thiruvananthapuram, India \\ *Corresponding Author \\ Dr Premlal A.P \\ Email: premlalap717@gmail.com
}

\begin{abstract}
Background: The main role of fine needle aspiration cytology (FNAC) lies in differentiating between a malignant and benign thyroid nodule. It greatly influences the treatment decision. The current study was undertaken to evaluate the cytology-histopathology correlation.

Materials and Methods: This is a retrospective study comparing cytology and corresponding histopathology report in 100 thyroid cases. The statistical analysis included false positive rate, false negative rate, sensitivity, specificity, positive predictive value, negative predictive value and accuracy.

Results: The sensitivity of FNAC is $92 \%$ in the present study and false negative occurred in $8 \%$ cases. The incidence of histopathologic surprise (as differentiated carcinoma thyroid) in nodular thyroid is $8 \%$. FNAC is a very powerful tool in the Preoperative investigator benign nodular-thyroid with a sensitivity of 92\%. Male: Female ratio in patients presenting with histologic surprise is 1:7. Age group which shows maximum chance for harbouring histologic surprise is between 25-30 years. Solitary nodular thyroid shows maximum false negative in FNAC followed by MNG.

Keywords: Cytology, FNAC, Thyroid, Thyroid cancer.
\end{abstract}

\section{Introduction}

Thyroid disease constitute a major bulk of cases attending any general surgery department of which thyroid carcinomas constitute a group of tumors that show considerable variability in biological behaviour histological appearance and response to therapy. Thyroid nodule is a common clinical problem. It can be palpated in $5 \%$ of individuals during thyroid examination and can be detected in up to $60 \%$ of people who undergo thyroid ultrasound. ${ }^{1,2}$ Most nodules are benign, but they are usually the first sign of thyroid cancer. ${ }^{1,3}$

Thyroid malignancies are rare, but its incidence has been increasing probably because of fairly accurate preoperative early detection. Most of them present as simple thyroid swelling very much similar to benign disease. Among the myriads of diseases affecting thyroid gland, 4-5\% 
are malignancies of which $90 \%$ are well differentiated cancers. Papillary carcinomas constitute $80-85 \%$ of these tumours.

Fine-needle aspiration cytology (FNAC) is an easy, cost-effective test for cancer diagnosis, and its use has markedly decreased the number of unnecessary thyroid surgeries. ${ }^{4}$ Although FNAC gives a preoperative diagnosis of malignancy with high sensitivity and specificity, few cases are missed in FNAC which are reported as histopathological surprise. This study is a sincere effort to find out the incidence of differentiated carcinoma thyroid as histological surprise in FNAC proved benign thyroid nodule.

\section{Aims of the Study}

1) To evaluate the incidence of differentiated carcinoma thyroid presenting as histological surprise in FNAC proved benign thyroid nodule.

2) To study the clinic-pathologic characteristics of patients presenting as histological surprise.

3) To assess the efficiency of FNAC as a preoperative diagnostic modality in the treatment of thyroid nodules.

\section{Materials and Methods}

This is a prospective observational study conducted at Government Medical College Thiruvananthapuram, in a one year period. Sample size was calculated as per statistical formula and was fixed at 100 . The patients attending the surgical OPD with thyroid swellings were subjected to physical examination and then subjected to cytologic analysis using FNAC. In all cases FNAC was done first using a $23 \mathrm{G}$ needle followed by surgery and histopathological examination. All patients underwent surgery in our department and the histopathology report of the thyroid specimen studied.

\section{Inclusion criteria}

Only the cases from which adequate material for FNAC was obtained were included in the study.

All the patients were above 13 years of age.
Only those patients who underwent thyroid surgery post FNAC

\section{Exclusion criteria}

Patients who have been referred with other causes of neck swelling

Age less than 13 years

Those patients who are not undergoing surgery.

After a detailed clinical examination along with making note of any doubtful sites on the thyroid gland the patient was investigated with a thyroid function test. After taking informed consent of the patient, the patient was subjected to the FNAC. Some of the smears were fixed in methanol and stained by Papanicolaou stain, the others were air dried and stained by May-Grunwald-Geimsa stain. No special stains were used.

\section{Observations}

\section{Gender Distribution of cases}

There were 91 females and 9 males in the study group (Figure 1)

\section{Figure 1}

\section{Gender Distribution}

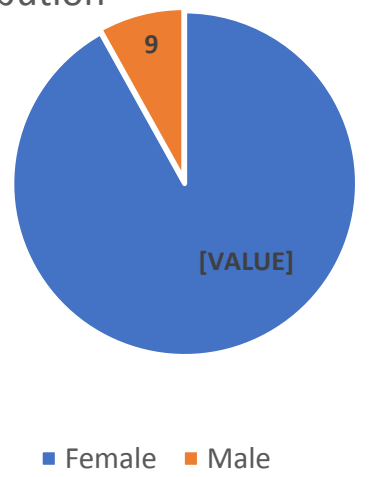

Male : female ratio 1:10.11

\section{Pre-Operative Diagnosis}

Of the 100 cases studied 46 cases had a preoperative diagnosis of multinodular goitre, 42 had a diagnosis of solitary nodular thyroid, 8 were toxic goitre and 4 Graves disease. (Figure 2) 
Figure 2 - Pre-Op Diagnosis

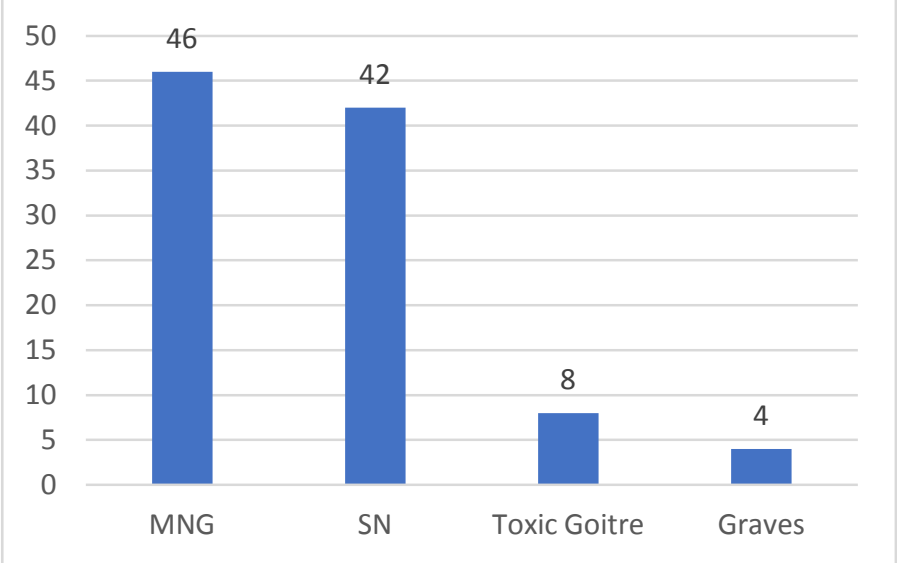

\section{Surgical Procedure}

Of the 100 cases - 42 underwent hemithyroidecotmy and 58 underwent subtotal thyroidectomy. None had total / near total thyroidectomy. (Figure 3)

Fig 3

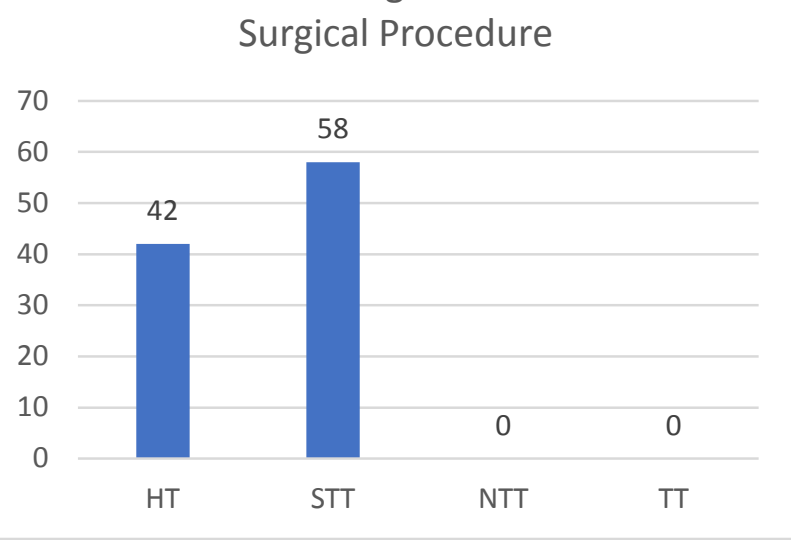

In our study our sensitivity of FNAC is $92 \%$. False negative rate is $8 \%$.

Of the 100 cases 92 cases had a similar FNAC and HPR. Remaining 8 cases presented as histological surprise in this cases they presented as papillary carcinoma thyroid. There was no case of follicular carcinoma, medullary carcinoma or anaplastic carcinoma. So this study showed $8 \%$ chance of histologic surprise - making the sensitivity of FNAC 92\%. (Figure 4)

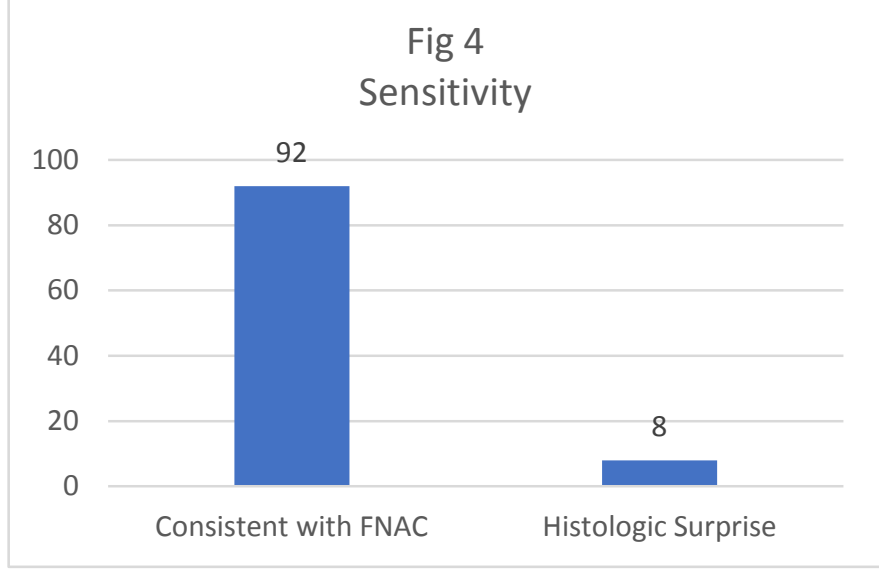

Of the 8 cases of papillary carcinoma thyroid presenting as histologic surprise, 3 had associated thyroiditis, 4 were follicular variant of papillary carcinoma and 1 was poorly differentiated variety. (figure 5)

Fig 5

Analysis of histologic surprise

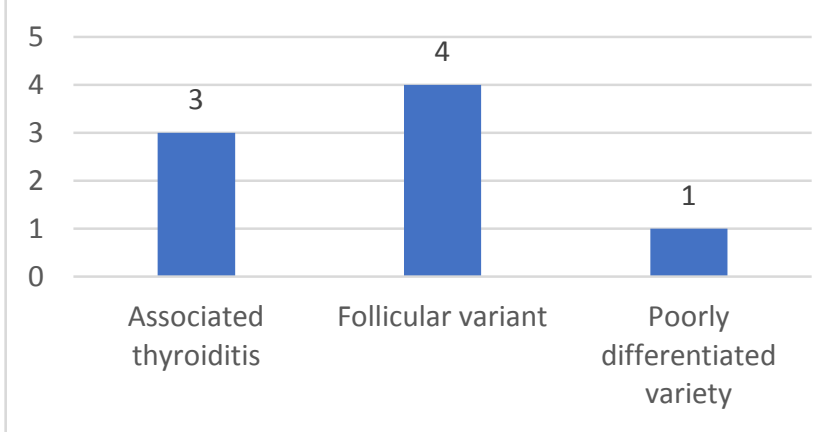

Of the 8 cases of papillary carcinoma thyroid presenting as histologic surprise, 3 had a preoperative diagnosis of multi nodular goiter, 4 had a diagnosis of solitary nodular thyroid and one was toxic goitre.

\section{Male female ratio in histologic surprise}

Of the 8 cases - there were 7 females and one male with male: female ratio of 1.7 (figure 6)

Fig 6

Male Female ratio

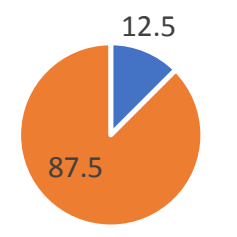

Male Female 
Age distribution of 8 cases presenting us histologic surprise maximum number of cases were seen between age group 25-30 years (Figure 7)

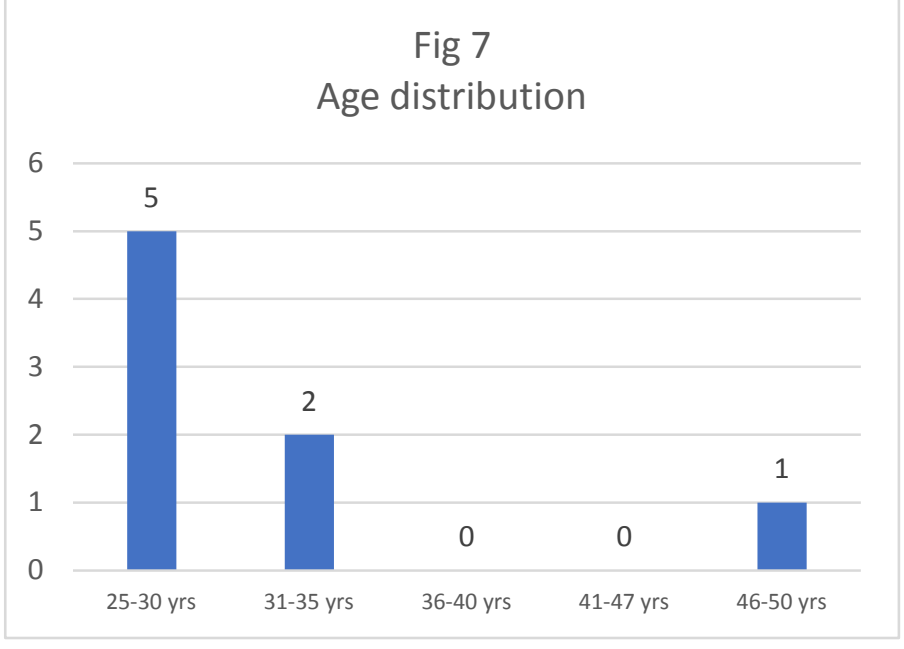

\section{Discussion}

The age and sex distributions of the patients in this study are similar to those reported by similar studies. ${ }^{2,3,5,6}$ In addition, the male to female ratio observed in this study for thyroid cancer (1:10.11) is in agreement with the fact that thyroid neoplasm is commoner among women. ${ }^{3}$

In this study, $8 \%$ of the cases were malignant. This finding is similar to Muratli et al., ${ }^{2}$ whose study reported a similar rate. FNAC is nowadays routinely performed for most cases of thyroid nodules. This has led to a reduction in the number of unnecessary surgeries and consequently to a rise in the percentage reported for malignancy. ${ }^{4}$

The percentages we observed for papillary carcinoma is $100 \%$, were higher compared to the figures reported in the previous studies on thyroid cancer. $^{2,3,5}$

Thyroid cancer comprises the bulk of the spectrum of endocrine malignancy. The preoperative diagnosis of malignancy can be done with FNAC which is having very high sensitivity.

Although FNAC is very sensitive, there are a few cases of false negative results which are proved by histopathological examination. Many studies were done all over the world to find out the effectiveness of FNAC as a preoperative diagnostic tool- all studies have shown some percentage of false negative cases which were reported as histologic surprise in histopathologic reports.

\section{FNAC diagnosis and histological diagnosis}

The diagnostic accuracy of Correlation between FNAC diagnosis and final histological diagnosis was $88.8 \%$ in a study by Basolo et al. ${ }^{7}$ Same was demonstrated as $90.9 \%$ in a study by Agarwal S. Study by Islam had accuracy of $94.44 \%$ and Sengupta A and group had accuracy of $98.31 \%$. In our study accuracy was $98.18 \% .{ }^{8.9 .10}$ In our study we correlated the cytological findings with HP findings for malignant lesions and found that the Sensitivity was $92 \%$, and false negative rate was $8 \%$

Although this is a study of a small group of patients the incidence of histopathologic suspires in a very serious issue in our day today practice$8 \%$ in this case. FNAC is still the investigation of choice for the preoperative diagnosis- but the most common cause for false- negative diagnosis consisted of sub optimal material and under diagnosis of papillary carcinoma due to cystic degeneration.

\section{Conclusion}

- The incidence of histopathologic surprise (as differentiated carcinoma thyroid) in nodular thyroid is $8 \%$.

- FNAC is a very powerful tool in the Preoperative investigator benign nodularthyroid with a sensitivity of $92 \%$.

- Male: Female ratio in patients presenting with histologic surprise is 1:7.

- Age group which shows maximum chance for harbouring histologic surprise is between 25-30 years.

- Solitary nodular thyroid shows maximum false negative in FNAC followed by MNG.

- In this study only papillary carcinoma thyroid was obtained as histopathologic surprise.

- Of the total 8 cases of papillary carcinoma3 had associated thyroiditis, 4 cases 
showed follicular variant and 1 case was poorly differentiated type.

- All patients undergoing lobectomy or hernithyroidectomy for Solitary Nodule Thyroid should be warned about the $8 \%$ chance of histopathologic surprise after obtaining hisiopathologic report.

- More work is needed in this held to improve the results of FNAC and thereby reducing the late or histopathologic surprise.

\section{Acknowledgments}

We would like to thank all staff in Pathology Department at Government Medical college, Thiruvananthapuram, for their cooperation and help in providing the data.

There are no conflicts of interest.

Funding: No funding sources

Conflict of interest: None declared

Ethical approval: The study was approved by the Institutional Ethics Committee

\section{References}

1. Esmaili HA, Taghipour H. Fine-needle aspiration in the diagnosis of thyroid diseases: An appraisal in our institution ISRN Pathology 2012. 2012912728.

2. Muratli A, Erdogan N, Sevim S, Unal I, Akyuz S. Diagnostic efficacy and importance of fine-needle aspiration cytology of thyroid nodules. J Cytol. 2014;31:73-8.[PMCID:

PMC4159900] [PubMed: 25210233]

3. Larijani B, Aghakhani S, Haghpanah V, Mosavi-Jarrahi A, Bastanhagh M. Review of thyroid cancer in Iran. Aus-Asian $\mathrm{J}$ Cancer. 2005;4:199-203.

4. Cibas ES, Ali SZ. NCI Thyroid FNA State of the Science Conference. The Bethesda system for reporting thyroid cytopathology Am J Clin Pathol. 2009;132:65865.[PubMed: 19846805]
5. Khayamzadeh $\mathrm{M}$, Khayamzadeh $\mathrm{M}$, Tadayon N, Salmanian R, Zham H, Razzaghi Z, et al. Survival of thyroid cancer and social determinants in Iran, 2001-2005. Asian Pac J Cancer Prev. 2011;12:95-8. [PubMed: 21517238]

6. Sinna EA, Ezzat N. Diagnostic accuracy of fine needle aspiration cytology in thyroid lesions. J Egypt Natl Canc Inst. 2012; 24:63-70. [PubMed: 23582597]

7. Basolo F, Ugolini C, Proietti A, Iacconi P, Berti P, Miccoli P. Role of frozen section associated with intraoperative cytology in comparison to FNA and FS alone in the management of thyroid nodules. European J Surgical Oncol. 2007;33(6):769-75.

8. Islam MS, Siddiquee BH, Akhtar N, Salam KS, Aktaruzzaman M. Comparative study of FNAC and histopathology in the diagnosis of thyroid swelling, Bangladesh J Otorhinolaryngol. 2010;16(1):35-43.

9. Sengupta A, Pal R, Kar S, Zaman FA, Sengupta S, Pal S. Fine needle aspiration cytology as the primary diagnostic tool in thyroid enlargement. J Nat Sci Biol Med. 2011;2(1):113-8.

10. Agarwal S. Diagnostic accuracy and role of fine needle aspiration cytology in management of thyroid nodules. J Surg Oncol. 1995:58(3):168-72. 